\title{
Full Endoscopic Technique for High-Grade Up-Migrated Lumbar Disk Herniation via a Translaminar Keyhole Approach: Preliminary Series and Technical Note
}

\author{
Guang-Xun Lin ${ }^{1} \quad$ Cheul-Woong Park ${ }^{2}$ Tsz-King Suen ${ }^{3}$ \\ ${ }^{1}$ Department of Orthopedics, The First Affiliated Hospital of Xiamen \\ University, Xiamen, PR China \\ 2 Department of Neurosurgery, Daejeon Woori Spine Hospital, \\ Daejeon, South Korea \\ ${ }^{3}$ Department of Orthopaedics \& Traumatology, Caritas Medical Centre, \\ Kowloon West Cluster Hospital Authority, Hong Kong, PR China \\ ${ }^{4}$ Department of Orthopaedics, Spine Unit, Queen Savang Vadhana \\ Memorial Hospital, Sriracha, Chonburi, Thailand \\ ${ }^{5}$ Department of Neurosurgery, Seoul St. Mary's Hospital, The \\ Catholic University of Korea, Seoul, South Korea
}

J Neurol Surg A 2020;81:379-386.

\author{
Address for correspondence jin-Sung Kim MD, PhD, Department of \\ Neurosurgery, Seoul St. Mary's Hospital, The Catholic University of \\ Korea, 222 Banpo-daero Seocho-gu, Seoul 06591, Republic of Korea \\ (e-mail: mdlukekim@gmail.com).
}

\begin{abstract}
Keywords

- translaminar

- full endoscopic lumbar diskectomy

- migrated disk herniation

- lumbar spine

Background and Study Aims Technically and anatomically, accessing a high-grade migrated lumbar disk herniation (LDH) using traditional full endoscopic lumbar diskectomy (FELD) approaches (either transforaminal or interlaminar) is challenging. The objective of this study was to present an effective and safe surgical approach for high-grade up-migrated LDH by translaminar FELD.

Patients and Methods Thirteen patients with soft high-grade up-migrated LDH treated with a translaminar FELD between May 2015 and July 2018 were reviewed in this study. Five of these patients had very high-grade up-migration. Clinical outcomes were assessed including preoperative and postoperative visual analog scale (VAS) for back and leg pain, Oswestry Disability Index (ODI), and MacNab criteria.

Results Overall, 7 of the 13 patients had disk-fragment migration at L4-L5: three at L5-S1, two at L3-L4, and the remaining one at L2-L3. In all cases, the highly upmigrated LDH was removed successfully through the translaminar approach, as confirmed by postoperative magnetic resonance imaging. The improvements of VAS for back and leg pain were $4.5 \pm 0.9$ to $1.3 \pm 1.3$ and $7.1 \pm 1.0$ to $1.6 \pm 0.7$, respectively (both $p<0.05$ ). The ODI decreased from preoperative $41.9 \pm 6.0$ to postoperative $13.0 \pm 4.1(p<0.05)$. According to the MacNab criteria, the satisfaction rate was $92.3 \%$ (excellent or good outcomes). None of the patients experienced any perioperative complications or recurrence during the follow-up period.

Conclusion Although full endoscopic technique via the translaminar keyhole route may not be used as a routine surgical approach, it could serve as a feasible alternative method for patients with highly up-migrated disk herniation.
\end{abstract}

received

February 26, 2019

accepted after revision

July 3, 2019

published online

February 11, 2020 (c) 2020 Georg Thieme Verlag KG

Stuttgart · New York
DOI https://doi.org/

10.1055/s-0039-1700574. ISSN 2193-6315. 


\section{Introduction}

With the development of endoscopic instruments and surgical approaches, percutaneous endoscopic spine surgery has become an alternative to open surgery, specifically in procedures involving decompression alone. ${ }^{1,2}$ For a minimally invasive surgery, full endoscopic lumbar diskectomy (FELD) was shown to result in reduced approach-related trauma, a shorter time of hospitalization, and faster recovery. ${ }^{2,3}$ However, up- or downmigration of the herniated disk occurs more frequently in the upper lumbar spine. ${ }^{4}$ In these patients, it was reported that the failure rates reach up to $20 \%$ using the traditional FELD (either transforaminal or interlaminar) approaches. ${ }^{5,6}$ The main reason for this increased risk of surgical failure is the relatively technically demanding and cumbersome process of debris removal, especially in cases of high-grade migration. Even for experienced endoscopic spine surgeons, treating highly migrated lumbar disk herniation (LDH) is a challenge.

Removing the migrated disk via limited laminar fenestration is theoretically possible and should be a more direct and less destructive approach. Under a microscope, the translaminar procedure of removing such a herniated disk requires a more traumatic approach with a larger opening of the spine due to the limited surgical vision around the corner. The use of an endoscope here is clearly advantageous compared with the use of a microscope. In the current study, we share our experience of applying a percutaneous endoscopic technique to high-grade up-migrated LDH through a translaminar route.

\section{Patients and Methods}

\section{Patients and Evaluation}

This retrospective study was approved by the institutional review board of our affiliated institution. Between May 2015 and July 2018, 146 patients underwent FELD for LDH. Among them, we reviewed 13 patients who underwent FELD using a translaminar approach for highly up-migrated or sequestrated soft disk herniation. The extent of migration is located above the halfway point of the infrapedicle level ( - Fig. 1 ) and located in the inner half of the foramen.

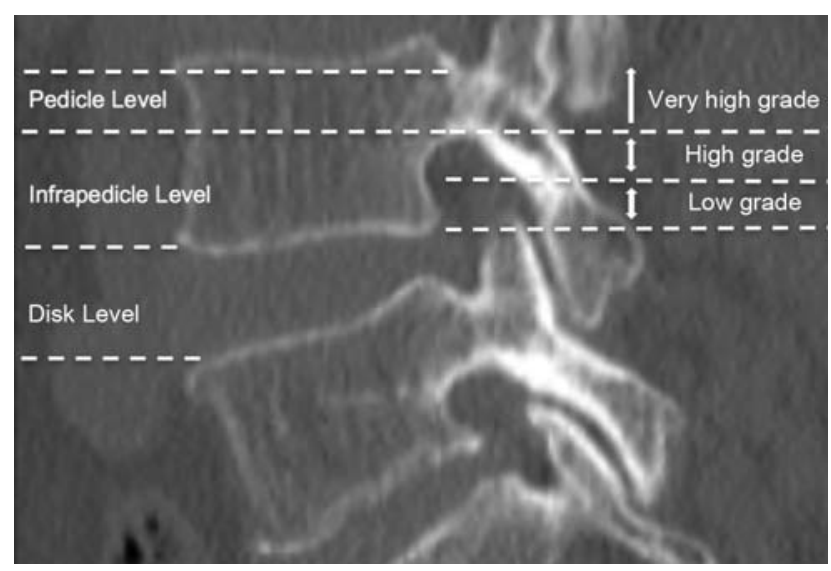

Fig. 1 Extent of migrated disk according to radiologic zones. Up-migration is divided into three zones: low grade, high grade, and very high grade.
The patients' data, including sex, age, operation level, and site of approach, were collected. Clinical outcomes using the visual analog scale and Oswestry Disability Index were charted at 1 month after surgery and at the last follow-up visit. A modified MacNab criterion was used to assess patient satisfaction. To confirm the adequate and complete removal of the disk, postoperative magnetic resonance imaging (MRI) and computed tomography (CT) were performed.

\section{Surgical Technique}

It was necessary with preoperative MRI and CT to confirm there was no calcification and no degenerative spinal stenosis (-Fig. 2a). All of the patients were under general anesthesia and placed on the radiolucent table in a prone position. The ideal fenestration trajectory is the area of the main migrated disk, lying exactly beneath the lamina, which could be targeted with preoperative axial images of MRI and CT scans ( - Fig. 2b). The level was confirmed under fluoroscopy. A needle was inserted into the surface of the lamina (-Fig. $\mathbf{2 c}$, d) and its position confirmed by fluoroscopy. Then the needle was replaced by a Kwire, and sequential dilators were inserted down to the surface of the lamina, docking the optimized ratio of large working channel $(5.6 \mathrm{~mm})$ to outside diameter $(10.5 \mathrm{~mm})$ for the least invasive traumatic access and a highly effective operation (-Fig. 2e, f). A 25-degree endoscopy was advanced through the working cannula (Vertebris Stenosis; RIWOspine, $\mathrm{GmbH}$, Knittlingen, Germany). Under high-resolution high-definition (HD) endoscopic visualization, the bony surface of the lamina was clearly exposed using a radiofrequency probe and forceps.

The next surgical step used an endoscopic high-speed diamond burr to drill a keyhole on the lamina (-Fig. $\mathbf{2 g}, \mathbf{h}$ ). The bottom of the keyhole was widened to provide adequate space. The drilling was performed carefully; the ligamentum flavum is very thin in this location, and sometimes the drill enters the epidural space directly. Following the removal of the ligamentum flavum, fat tissue, and axillary veins, the migrated disks were usually identified. Endoscopic forceps (-Fig. 2i) and hooks ( $\mathbf{- F i g . ~ 2 j , ~} \mathbf{k}$ ) were used to remove migrated or sequestrated disks, and using the probe, the complete decompression was verified when the nerve root was seen floating freely in the epidural space ( - Video 1 ). The epidural bleeding was controlled with the use of Gelfoam (Pfizer, New York, New York, United States) that was removed 2 to 3 minutes later; the endoscopic procedures were performed for the removal of migrated disk fragments, and the bulging components of the disk level were not removed. The patient's outcome was not influenced by the asymptomatic bulging disk, and it would be reabsorbed with time.

\section{Video 1}

Full endoscopic technique for high-grade up-migrated lumbar disk herniation via a translaminar keyhole approach. Online content including video sequences viewable at: www.thieme-connect.com/products/ ejournals/html/10.1055/s-0039-1700574. 


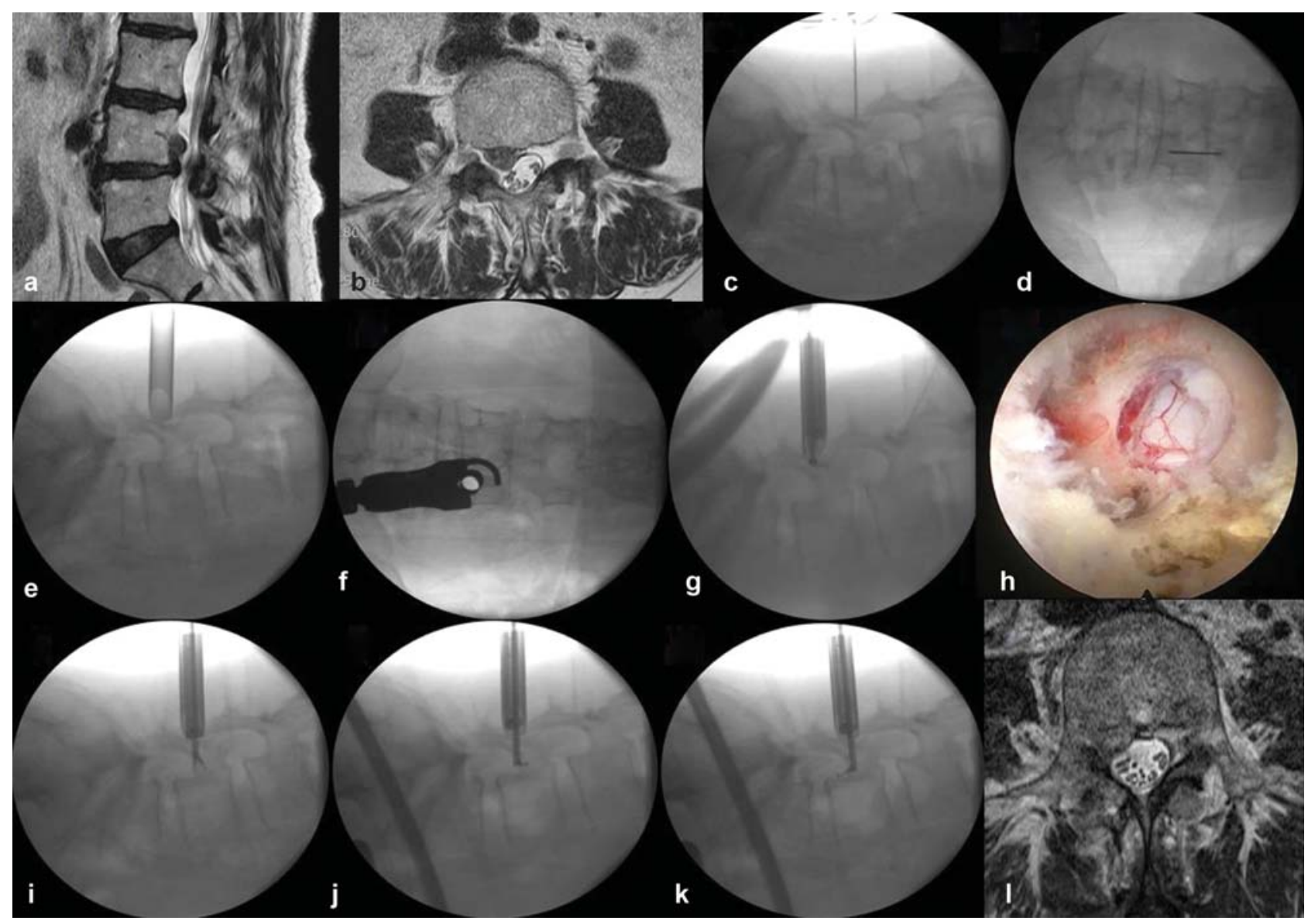

Fig. 2 L4-L5 up-migrated disk fragment. (a) Preoperative sagittal T2 magnetic resonance imaging (MRI) shows high-grade up-migrated disk from L4 / L5. (b) Preoperative axial T2 MRI shows right up-migrated disk located at axilla site of L4 root. (c) Lateral fluoroscopic view of the K-wire positioned on the surface of the lamina of L4. (d) Anteroposterior (AP) fluoroscopic view of the K-wire positioned on the surface of the lamina of L4. (e) Lateral fluoroscopic view of the working cannula introduced to the surface of the lamina. (f) AP fluoroscopic view of the working cannula introduced to the surface of the lamina. (g) Lateral fluoroscopic view of the diamond burr used to drill a keyhole on the lamina. (h) Endoscopic view of the bony keyhole made in the lamina. The dural sac can be observed at the bottom of the keyhole. (i) Lateral fluoroscopic view of the endoscopic grasping forceps removing the up-migrated disk. (j, k) Lateral fluoroscopic view of the endoscopic 90-degree angle blunt hook exploring the root and ventral aspect of the dural sac. (I) Postoperative axial T2 MRI shows the disappearance of the herniated disk.

\section{Results}

Six female patients and seven male patients underwent operations using the endoscopic translaminar technique in our hospital ( - Table 1 ). The mean age was 49.8 years (range: 29-68 years). The mean follow-up was 20 months (range: 441 months). The mean operative time was 79.2 minutes (range: 50-110 minutes). The mean estimated blood loss was $29.2 \mathrm{~mL}$ (range: $20-50 \mathrm{~mL}$ ). All patients showed highgrade up-migration of LDH, and five of them were very highly up-migrated (above the pedicle level).

The clinical outcomes were significantly improved after surgery ( $p<0.05$; - Table 2 ). We observed excellent or good outcomes in $92.3 \%$ of patients following surgery according to the MacNab criteria. No perioperative complications or recurrence occurred in any patients during the follow-up period.

In all cases, the postoperative MRI demonstrated complete decompression of the nerve root and dural sac. On the postoperative reconstructed $\mathrm{CT}$ images, the bone resection border of the lamina and the translaminar channel is clearly shown (-Fig. 3).

\section{Case Studies}

\section{Case 1}

A 57-year-old male patient complained of severe left leg pain and weakness (hip flexion and knee extension, grade IV) that started 1 month before his visit. MRI showed a very highgrade up-migrated disk from L2/L3 (-Fig. 4a, b), almost above the pedicle level. Therefore, we decided to approach through the lamina to remove the migrated disk ( - Fig. 4c, d). One-day postoperative MRI revealed the successful removal of the very highly migrated disk ( - Fig. 4e, f). Postoperatively, the patient achieved an excellent outcome, and the weakness was completely eradicated 2 months after surgery.

\section{Case 2}

A 34-year-old male patient complained of left leg pain and weakness (ankle dorsiflexion, grade IV; great toe extension, grade III) combined with left whole-foot numbness that started 3 weeks before surgery after a lifting injury. After 2 weeks of conservative treatment, the patient's symptoms did not improve. Preoperative MRI showed a high-grade up-migrated 
Table 1 Characteristics of 13 patients

\begin{tabular}{|l|l|l|l|l|l|l|l|l|l|}
\hline No. & Age, y & Sex & F/U, mo & Level & Extent & Side & $\begin{array}{l}\text { Operation } \\
\text { time, } \text { min }\end{array}$ & $\begin{array}{l}\text { Estimated } \\
\text { blood loss, } \mathrm{mL}\end{array}$ & MacNab criteria \\
\hline 1 & 68 & F & 31 & L4-L5 & H & Right & 110 & 40 & Good \\
\hline 2 & 57 & M & 15 & L2-L3 & VH & Left & 95 & 50 & Excellent \\
\hline 3 & 34 & M & 29 & L5-S1 & H & Left & 80 & 20 & Excellent \\
\hline 4 & 67 & F & 41 & L4-L5 & H & Left & 55 & 20 & Excellent \\
\hline 5 & 43 & M & 34 & L4-L5 & VH & Right & 95 & 30 & Fair \\
\hline 6 & 29 & M & 17 & L4-L5 & VH & Left & 65 & 40 & Good \\
\hline 7 & 30 & M & 12 & L4-L5 & H & Right & 80 & 30 & Good \\
\hline 8 & 39 & M & 40 & L5-S1 & H & Right & 110 & 25 & Excellent \\
\hline 9 & 49 & F & 8 & L4-L5 & H & Right & 60 & 20 & Excellent \\
\hline 10 & 62 & F & 8 & L3-L4 & VH & Right & 50 & 25 & Excellent \\
\hline 11 & 55 & F & 10 & L4-L5 & VH & Right & 65 & 20 & Good \\
\hline 12 & 66 & M & 11 & L3-L4 & H & Right & 55 & 30 & Good \\
\hline 13 & 68 & F & 4 & L5-S1 & H & Right & 110 & 30 & \\
\hline
\end{tabular}

Abbreviations: $\mathrm{F} / \mathrm{U}$, follow-up; $\mathrm{H}$, high-grade up-migration; $\mathrm{VH}$, very high-grade up-migration.

Table 2 Clinical outcomes in 13 patients

\begin{tabular}{|c|c|c|c|c|c|c|}
\hline \multirow[t]{2}{*}{ Parameters } & \multicolumn{3}{|l|}{$N=13$} & \multicolumn{3}{|c|}{$p$ value: ANOVA test } \\
\hline & Preoperative & 1-mo postoperative & Last $\mathrm{F} / \mathrm{U}$ & $\begin{array}{l}\text { Preoperative } \\
\text { vs. } 1 \text { mo }\end{array}$ & $\begin{array}{l}\text { Preoperative } \\
\text { vs. last }\end{array}$ & $\begin{array}{l}1 \text { mo } \\
\text { vs. last }\end{array}$ \\
\hline VAS back (range) & $4.5 \pm 0.9(3-6)$ & $2.5 \pm 0.5(2-3)$ & $1.3 \pm 1.3(0-3)$ & \multirow{3}{*}{\multicolumn{3}{|c|}{$<0.05$}} \\
\hline VAS leg (range) & $7.1 \pm 1.0(5-9)$ & $2.7 \pm 0.6(2-4)$ & $1.6 \pm 0.7(0-2)$ & & & \\
\hline ODI (range) & $41.9 \pm 6.0(32-52)$ & $24.0 \pm 4.0(18-30)$ & $13.0 \pm 4.1(8-20)$ & & & \\
\hline
\end{tabular}

Abbreviations: ANOVA, analysis of variance; F/U, follow-up; ODI, Oswestry Disability Index; VAS, visual analog scale.

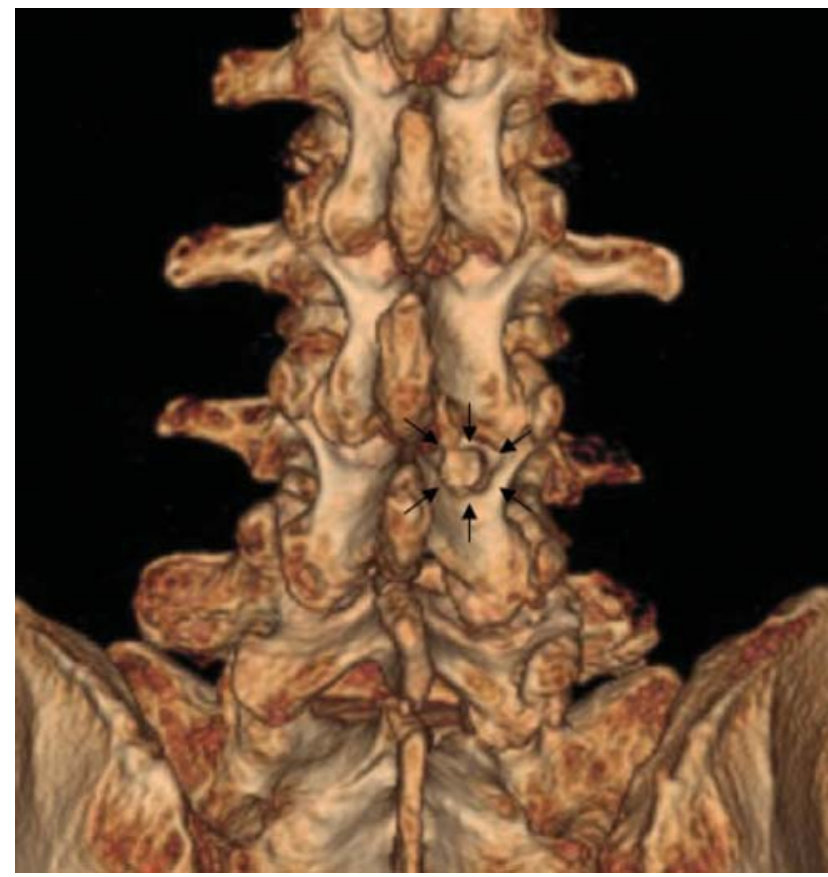

Fig. 3 Postoperative computed tomography scan shows the bony keyhole (black arrows).
LDH from L5/S1 (- Fig. 5a, b). We performed a translaminar FELD removal of the migrated disk through a keyhole. One-day postoperative MRI showed the main migrated disk fragments had disappeared, but a small number of residual masses were visible ( $\mathbf{- F i g . ~ 5 c , ~ d ) . ~ O n e - y e a r ~ p o s t o p e r a t i v e ~ M R I ~ s h o w e d ~ a n ~}$ asymptomatic residual mass reabsorbed with time( - Fig. 5e, f). The patient achieved an excellent clinical outcome, and the weakness was completely gone at 4 months after surgery.

\section{Discussion}

Disk fragments can penetrate into the posterior longitudinal ligament and migrate cranial or caudal of the anterior epidural space, reportedly at an incidence of 35 to $72 \%$. 3,7 In general, migration and sequestration are usually located on the pars interarticular medial to the pedicle and may reach the medial level of the vertebra. ${ }^{7,8}$ In particular, upmigrated LDH is mostly sequestrated and discontinuous from the origin intervertebral disk, and it is sometimes separated into multiple pieces. ${ }^{9}$

The removal of these migrated multiple fragments by the conventional surgical technique, and specifically the removal of the sequestrated fragments, requires extensive resection of 


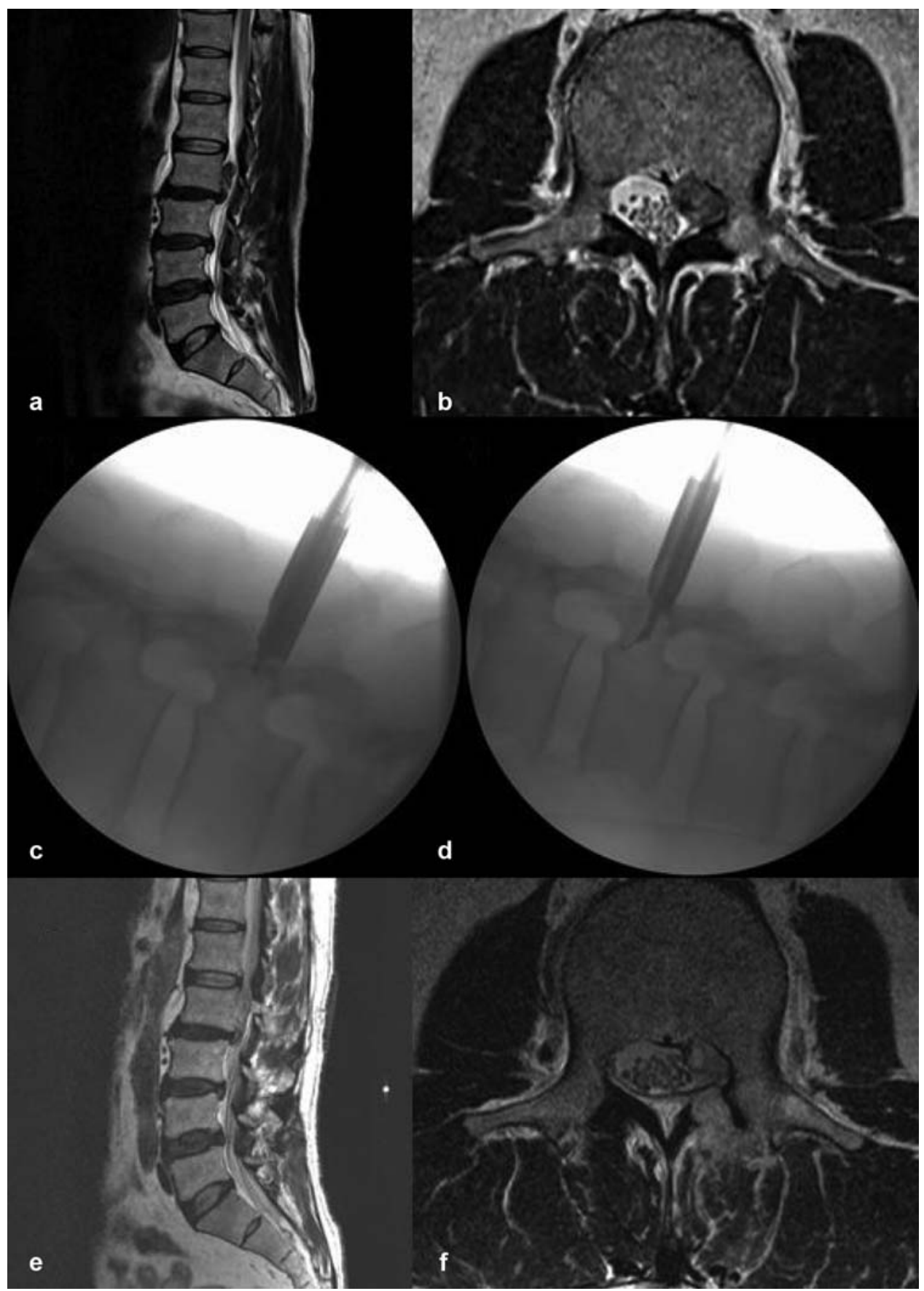

Fig. 4 (a, b) Preoperative sagittal and axial T2 magnetic resonance imaging (MRI) shows very high-grade up-migrated disk from L2 / L3. (c, d) Removal of the up-migrated disk through the translaminar approach. (e, f) One-day postoperative sagittal and axial T2 MRI shows successful removal of the migrated disk.

the lamina and the facet joints in particular, which may lead to postoperative instability and other complications. ${ }^{10,11}$ It may be possible to tailor the bony opening to minimize the likelihood of postoperative instability, especially with an experienced surgeon using a microscope. ${ }^{10,12}$ However, up- and downward migrated sequestrations are difficult to reach and remove completely with a microscope, and they cannot be examined around the corner. Here the use of high-quality endoscopes provides better imaging and more precise control of surgery (complete removal of the herniated disk and avoidance of damages because the area of the migrated disk is fully visualized, in full HD quality, and very bright illumination through the endoscope).

Several new surgical techniques have been designed to reach the migrated LDHs. Ahn et $\mathrm{al}^{13}$ reported that 13 patients ( 8 with up-migration and 5 with down-migration) who underwent transforaminal FELD using navigable instruments had excellent or good outcomes in $85 \%$ of cases, and symptom improvements were observed in $92 \%$ of cases 1 year after surgery. In addition, Choi et $\mathrm{al}^{8}$ described the use of a transforaminal epiduroscopic technique for migrated LDH in 59 patients. For highly up-migrated LDH, they started 


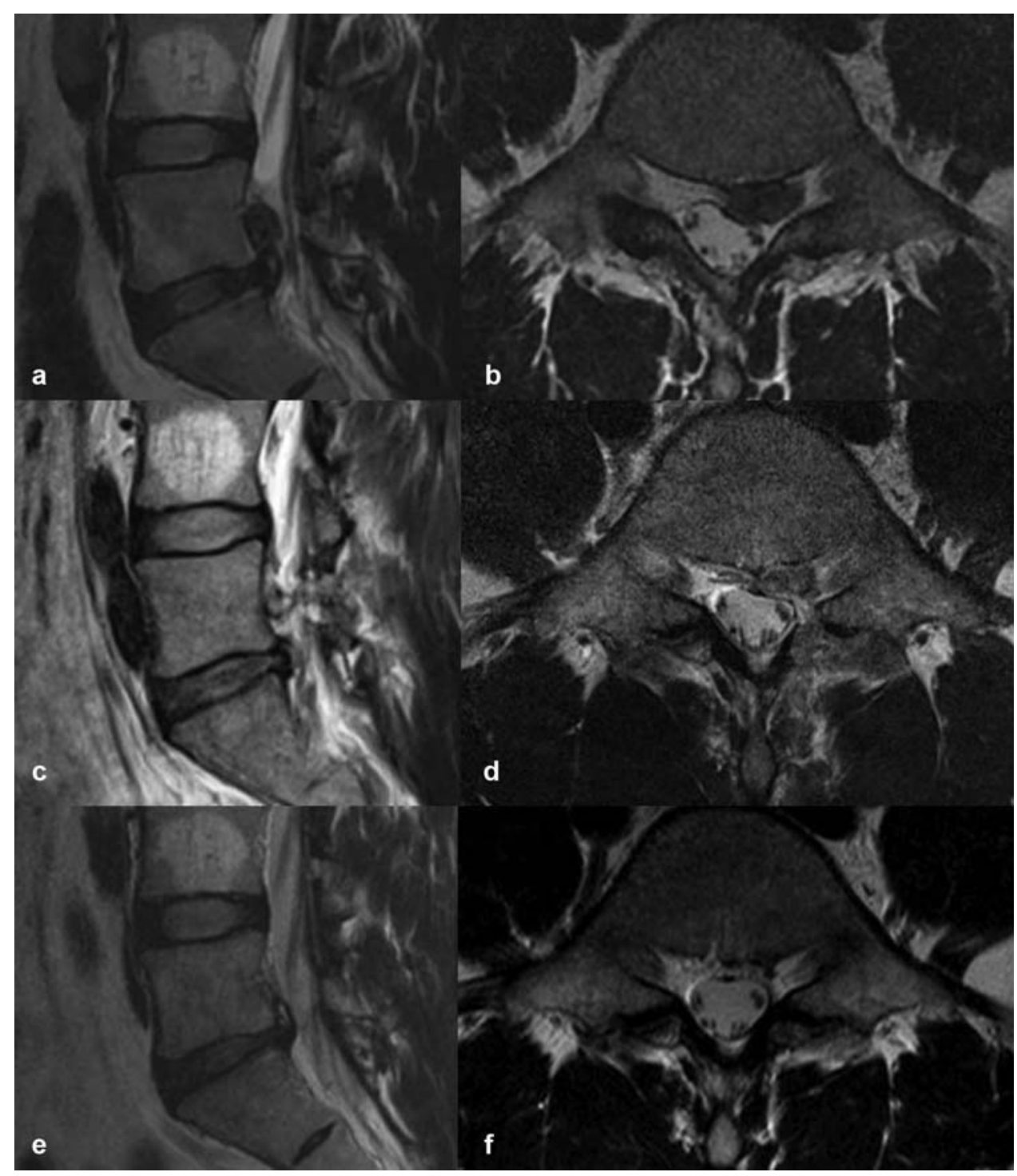

Fig. 5 (a, b) Preoperative sagittal and axial T2 magnetic resonance imaging (MRI) shows high-grade up-migrated disk from L5 to S1. (c, d) Oneday postoperative sagittal and axial T2 MRI shows successful removal of the main migrated disk, but a small number of residual fragments were visible. (e, f) One-year postoperative sagittal and axial MRI shows asymptomatic residual herniation reabsorbed with time.

from the epidural space of the disk level and gradually shifted upward to remove the disk, and the authors also mentioned that if the migrated LDH was multifragmented, additional foraminoplasty was required to remove those fragments completely. This may indirectly cause more bleeding and neural injuries. Likewise, prolonged procedural time and far distance manipulation in the canal may increase unpredictable complications. After the mean follow-up of 14.5 months, they found patients with high-grade migration of LDH had inferior outcomes compared with patients with low-grade migration of LDH.

In contrast, the interlaminar approach can directly access the ends of the fragments, meaning it is similar to standard open diskectomy because it is not limited by a high iliac crest. Kim et al ${ }^{9}$ reported 17 patients ( 6 with up-migration and 11 with down-migration) who underwent interlaminar FELD and obtained successful outcomes in $83 \%$ of cases after surgery. However, if there was no large interlaminar window for endoscopic access, a laminotomy was necessary with was performed using an endoscopic drill or punch. Under the endoscopic view, this may be a time-consuming task. Moreover, Ruetten et $\mathrm{al}^{14}$ suggested that when the fragments migrate beyond the upper middle of the vertebral body, the application of the interlaminar FELD is likely not conducive. Another recent article retrospectively reviewed a total of 149 patients who received FELD. ${ }^{15}$ The authors mentioned that neither the transforaminal nor the interlaminar technique is suitable for up-migration of the disk located above or downmigration below the pedicle level.

Translaminar FELD is similar to interlaminar FELD. In 2012, Dezawa et al ${ }^{16}$ described the use of the translaminar FELD technique in nine patients with migrated disk (located at the part of the subarticular and foraminal region). However, the authors did not mention the direction and extent of the migration. No complications were found, and the clinical outcomes were acceptable. In 2016, one article reported the 
use of the translaminar FELD technique for highly downmigrated LDH in seven patients; five of them were revision surgery. ${ }^{17}$ They described five patients who received a transforaminal or interlaminar FELD for a down-migrated disk and in which they did not achieve complete decompression in their initial operation. Using the translaminar FELD technique, the authors could completely remove the remaining disk in revision surgery and achieve satisfactory postoperative clinical results. Most recently, another article described the use of the "translaminar" technique in 11 patients with highly up-migrated LDH. ${ }^{18}$ In actuality, their technique was not a strictly translaminar approach. The authors made a beveled tunnel on the lateral bone of the laminar using a trephine to remove the migration.

To obtain adequate and direct exposure of the main migrated fragments, we used a real translaminar keyhole approach in patients with high-grade up-migrated LDH. These are the advantages of using the translaminar endoscopic technique:

1. It is an ultimately a minimally invasive procedure with minimal disruption of the soft tissues and posterior bone structures (lamina and facet joints). Therefore, iatrogenic instability can be avoided.

2. The shorter procedural time and shorter distance of manipulation in the canal may reduce the potential risk of complications.

3. It reduces epidural scar formation.

4. It enables patients to resume work as soon as possible.

However, this approach has some limitations that are worth mentioning:

1. When the sequestrated fragments were broken into multiple pieces, after removal of the main fragments, a small part of the hidden fragment may still exist. However, some surgeons have mentioned that fragmentectomy could be acceptable because the residual disk could be resorbed over time. ${ }^{5,8,9}$ Practically, we also routinely perform mainly fragmentectomy in transforaminal or interlaminar FELD.

2. Sometimes, the migrated fragment is still consistent with the disk of origin. At this point, it is not easy to remove the pathology at the disc level through the translaminar keyhole. In the current study, one patient complained of only a fair postoperative outcome because of the incompletely removed herniation at the disk level.

3. The indications of the translaminar approach are limited: (a) It is only an alternative for highly migrated fragments that cannot be removed by the transforaminal or the interlaminar approach. In our study, the extent of migration was relatively high; 5 of 13 cases even exceeded the pedicle level. (b) It is applied to a migrated fragment located at the axilla site of the exiting nerve root and blocked by the lamina. If migrated fragments are located at the shoulder part of the upper nerve root, we recommend that another transpedicular approach be used. ${ }^{19}$

4. Care should be taken to avoid damage to dura or nerve root when the lamina is opened with a drilling burr because at this region, it lacks the protection of the ligamentum flavum.

This study had some limitations. It was a retrospective study with a small sample size and a short follow-up period. Nevertheless, we wanted to share our own experience with high-grade up-migrated LDH.

\section{Conclusion}

In patients with high-grade up-migrated sequestrations, which are difficult to reach and to remove completely with a microscope, the use of a high-quality endoscope and endoscope instruments allows complete removal of the herniated disk and avoidance of damages because the area of the migrated disk is fully visualized. The selection of the surgical approach is based on the direction and extent of migration of LDH. The translaminar FELD approach could be considered a feasible alternative surgical option for highgrade up-migrated LDH. This endoscopic technique is a lowinvasive method that allows for full visual control of the anatomy as well as neurosurgical manipulation around the corner.

\section{Conflict of Interest \\ None declared.}

\section{References}

1 Lee DY, Ahn Y, Lee SH. Percutaneous endoscopic lumbar discectomy for adolescent lumbar disc herniation: surgical outcomes in 46 consecutive patients. Mt Sinai J Med 2006;73(06):864-870

2 Brouwer PA, Brand R, van den Akker-van Marle ME, et al. Percutaneous laser disc decompression versus conventional microdiscectomy in sciatica: a randomized controlled trial. Spine J 2015;15(05): 857-865

3 Pan L, Zhang P, Yin Q. Comparison of tissue damages caused by endoscopic lumbar discectomy and traditional lumbar discectomy: a randomised controlled trial. Int J Surg 2014;12(05): 534-537

4 Ebeling U, Reulen HJ. Are there typical localisations of lumbar disc herniations? A prospective study. Acta Neurochir (Wien) 1992; 117(3-4):143-148

5 Lee S, Kim SK, Lee SH, et al. Percutaneous endoscopic lumbar discectomy for migrated disc herniation: classification of disc migration and surgical approaches. Eur Spine J 2007;16(03): 431-437

6 Lee SH, Kang BU, Ahn Y, et al. Operative failure of percutaneous endoscopic lumbar discectomy: a radiologic analysis of 55 cases. Spine 2006;31(10):E285-E290

7 Schellinger D, Manz HJ, Vidic B, et al. Disk fragment migration. Radiology 1990;175(03):831-836

8 Choi G, Lee SH, Lokhande P, et al. Percutaneous endoscopic approach for highly migrated intracanal disc herniations by foraminoplastic technique using rigid working channel endoscope. Spine 2008;33(15):E508-E515

9 Kim CH, Chung CK, Woo JW. Surgical outcome of percutaneous endoscopic interlaminar lumbar discectomy for highly migrated disk herniation. Clin Spine Surg 2016;29(05):E259-E266

10 Bernucci C, Giovanelli M. Translaminar microsurgical approach for lumbar herniated nucleus pulposus (HNP) in the "hidden zone": clinical and radiologic results in a series of 24 patients. Spine 2007;32(02):281-284 
11 Osman SG, Nibu K, Panjabi MM, Marsolais EB, Chaudhary R. Transforaminal and posterior decompressions of the lumbar spine. A comparative study of stability and intervertebral foramen area. Spine 1997;22(15):1690-1695

12 Reinshagen C, Ruess D, Molcanyi M, et al. A novel translaminar crossover approach for pathologies in the lumbar hidden zone. J Clin Neurosci 2015;22(06):1030-1035

13 Ahn Y, Jang IT, Kim WK. Transforaminal percutaneous endoscopic lumbar discectomy for very high-grade migrated disc herniation. Clin Neurol Neurosurg 2016;147:11-17

14 Ruetten S, Komp M, Merk H, Godolias G. Recurrent lumbar disc herniation after conventional discectomy: a prospective, randomized study comparing full-endoscopic interlaminar and transforaminal versus microsurgical revision. JSpinal Disord Tech 2009;22(02):122-129

15 Choi KC, Lee DC, Shim HK, Shin SH, Park CK. A strategy of percutaneous endoscopic lumbar discectomy for migrated disc herniation. World Neurosurg 2017;99:259-266
16 Dezawa A, Mikami H, Sairyo K. Percutaneous endoscopic translaminar approach for herniated nucleus pulposus in the hidden zone of the lumbar spine. Asian J Endosc Surg 2012;5(04): 200-203

17 Du J, Tang X, Jing X, Li N, Wang Y, Zhang X. Outcomes of percutaneous endoscopic lumbar discectomy via a translaminar approach, especially for soft, highly down-migrated lumbar disc herniation. Int Orthop 2016;40(06):1247-1252

18 Xin Z, Liao W, Ao J, et al. A modified translaminar osseous channel-assisted percutaneous endoscopic lumbar discectomy for highly migrated and sequestrated disc herniations of the upper lumbar: clinical outcomes, surgical indications, and technical considerations. BioMed Res Int 2017; 2017:3069575

19 Quillo-Olvera J, Akbary K, Kim JS. Percutaneous endoscopic transpedicular approach for high-grade down-migrated lumbar disc herniations. Acta Neurochir (Wien) 2018;160(08): 1603-1607 